International Journal of Pure and Applied Mathematics

Volume 113 No. 1 2017, 167-179

ISSN: 1311-8080 (printed version); ISSN: 1314-3395 (on-line version)

url: http://www.ijpam.eu

doi: 10.12732/ijpam.v113i1.15

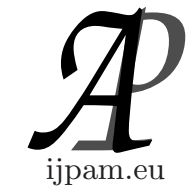

\title{
COMMON FIXED POINT THEOREM FOR MULTI-VALUED MAPPINGS ON B-METRIC SPACES
}

\author{
Chanakan Jinakul ${ }^{1}$, Araya Wiwatwanich ${ }^{2}$, Annop Kaewkhao ${ }^{3} \S$ \\ 1,2,3 Department of Mathematics \\ Faculty of Science \\ Burapha University \\ Chonburi Province 20131, THAILAND \\ Centre of Excellence in Mathematics \\ PERDO, CHE, Thailand
}

\begin{abstract}
In this paper, we prove a common fixed point theorem for multi-valued mappings in complete b-metric spaces. The conditions for existence of a common fixed point had been investigated. The main result can be regarded as a generalization of previous results in complete metric space.
\end{abstract}

AMS Subject Classification: 47H10, 54H 25

Key Words: common fixed point, multi-valued mapping, b-metric space

\section{Introduction}

Fixed point theory is one of cornerstones in the development of mathematics. It has been applied in physical sciences, computing sciences, engineering and so on. In this study, fixed point theory has started by letting $X$ be a nonempty set and $T: X \rightarrow X$. We say that $x \in X$ is a fixed point of $X$ if $T(x)=x$. In 1922, Banach [1] discovered that any contraction mapping in a complete metric space has a unique fixed point. The theorem has been further developed in different directions by Kannan [2] and [3], Kirk, Srinivasan, Veeramani [4], and many others.

$\begin{array}{ll}\text { Received: } & \text { January 20, } 2017 \\ \text { Revised: } & \text { February 21, } 2017 \\ \text { Published: } & \text { February 28, } 2017 \\ \S_{\text {}} \text { Correspondence author }\end{array}$

(c) 2017 Academic Publications, Ltd. url: www.acadpubl.eu 
In 1969 Nadler first initiated the study of fixed point theorems for the multi-valued contraction mappings. Let $(X, d)$ be a complete metric space, $C B(X)$ be a collection of all nonempty closed and bounded subsets of $X$ and $T: X \rightarrow C B(X)$ is called a multi-valued mapping. The original theorem on multi-valued fixed point is stated in Theorem 1.

Theorem 1. ([5]) Let $(X, d)$ be a complete metric space, and let $T: X \longrightarrow$ $C B(X)$ be a multi-valued contraction mappings, i.e., there exists a constant $a \in[0,1)$ such that

$$
H(T x, T y) \leq a d(x, y),
$$

for all $x, y \in X$. Then $T$ has a fixed point.

In addition, many researchers such as Kannan [2] and [3], Gupta, Srivastava [6] and Jungck [7] also studied the existence of a common fixed point multivalued mappings. According to those research, an element $z$ of $X$ is called a common fixed point of $T$ and $S$ if $T z=S z=z$. The study of a common fixed point for multi-valued mappings $T, S: X \longrightarrow C B(X)$ has been widely developed and extended by the following researchers. On complete metric space has been proved by Lai-jiu Lin and Sung-Yu Wang [8] have proved the following theorem.

Theorem 2. ([8]) Let $(X, d)$ be a complete metric space, and let $S, T$ : $X \longrightarrow C B(X)$ be a multi-valued mappings. If there exists $r \in[0,1)$ such that

$$
H(T x, S y) \leq r d(x, T y)
$$

for all $x, y \in X$. Then $F(T)=F(S) \neq \phi$ and $T x=S x=F(T)$ for all $x \in F(T)$.

The concept of b-metric space was introduced by Czerwik [9] in 1993. Some examples of b-metric space and some fixed point theorems in b-metric space can be found in Aydi, Bota, Karapinar \& Mitrovic [10], Bota [11], etc. Particulary, Reich [12], Mizoguchi-Takahashi [13] developed, extended and improved some fixed point theories of single-valued mapping and multi-valued mapping in bmetric space. The study of a common fixed point for multi-valued mappings has been widely developed and extended by the following researchers. On complete metric space, Lia-Jiu Lin and Sung-Yu Wang [8] have proved the theorem.

The concept of b-metric was introduced by Czerwik [14]. We present some definition in b-metric space as follows.

Definition 3. ([14]) Let $X$ be a nonempty set and $s \geq 1$ be a given real number. A function $d: X \times X \longrightarrow[0, \infty)$ is called a b-metric if and only if for all $x, y, z \in X$, the following conditions are satisfied:

(1) $d(x, y)=0$ if and only if $x=y$ 
(2) $d(x, y)=d(y, x)$

(3) $d(x, z) \leq s[d(x, y)+d(y, z)]$.

The triplet $(X, d, s)$ is called a b-metric space.

Definition 4. ([15]) Let $(X, d, s)$ be a b-metric space. The sequence $\left\{x_{n}\right\}$ in $X$ is called:

(1) Convergent if and only if for all $\varepsilon>0$, there exists $k \in \mathbb{N}$ we have such that $d\left(x_{n}, x\right)<\varepsilon$ for all $n \geq k$. In this case, we write $\lim _{n \rightarrow \infty} d\left(x_{n}, x\right)=0$.

(2) Cauchy sequence if and only if for all $\varepsilon>0$, there exists $k \in \mathbb{N}$ such that $d\left(x_{m}, x_{n}\right)<\varepsilon$ for all $m, n \geq k$.

Definition 5. ([15]) Let $(X, d, s)$ be a b-metric space. A subset $Y \subseteq X$ is called:

(1) closed if and only if for each sequence $\left\{x_{n}\right\}$ in $Y$, which converges to an element $x$, we have $x \in Y$.

(2) compact if and only if for every sequence of element in $Y$ there exists a subsequence that converges to an element in $\mathrm{Y}$.

(3) bounded if and only if $\sup \{d(x, y): x, y \in Y\}<\infty$.

Definition 6. The b-metric space $(X, d, s)$ is said to be complete if and only if every Cauchy sequence converges to some $x \in X$.

Next, we recall that $C B(X)$ on b-metric space denotes the class of nonempty closed and bounded subset of $X$. For $A, B \in C B(X)$, define the function $H: C B(X) \times C B(X) \longrightarrow[0, \infty)$ by

$$
H(A, B)=\max \{\delta(A, B), \delta(B, A)\}
$$

where

$$
\begin{aligned}
& \delta(A, B)=\sup \{d(a, B), a \in A\} \\
& \delta(B, A)=\sup \{d(b, A), b \in B\}
\end{aligned}
$$

with

$$
d(a, B)=\inf \{d(a, x), x \in B\}
$$

and

$$
d(A, B)=\inf \{d(a, b), a \in A, b \in B\} .
$$

By definition of the function $H$, we can easily proof the following lemma.

Lemma 7. Let $(X, d, s)$ be a b-metric space. For any $A, B, C \in C B(X)$ and any $x, y \in X$, the following statements are true:

(1) $d(x, B) \leq d(x, b)$, for any $b \in B$;

(2) $\delta(A, B) \leq H(A, B)$; 
(3) $d(x, B) \leq H(A, B)$, for any $x \in A$;

(4) $H(A, B)=0$ if and only if $A=B$;

(5) $H(A, B)=H(B, A)$;

(6) $H(A, C) \leq s(H(A, B)+H(B, C))$;

(7) $d(x, A) \leq s(d(x, y)+d(y, A))$.

Note that, we can see that $(C B(X), H, s)$ is a b-metric space and $H$ is called the Hausdorff b-metric induced by the b-metric $d$.

The following lemmas are useful for some of the proofs in the main result.

Lemma 8. Let $(X, d, s)$ be a b-metric space and $B$ be a nonempty closed subset of $X$ and $d(x, B)=0$ then $x \in B$.

Proof. By the definition of $d(a, B)$, for each $n_{0} \in \mathbb{N}$, we have $b_{n} \in B$ such that $d\left(a, b_{n_{0}}\right)<\frac{1}{n_{0}}$. Now, we have a sequence $\left\{b_{n}\right\}$ in $B$ such that $d\left(a, b_{n}\right)<$ $\frac{1}{n}, \forall n \in \mathbb{N}$. Next, we show that $\lim _{n \rightarrow \infty} d\left(a, b_{n}\right)=0$. Let $\varepsilon>0$, by the Archimedean property there exists $N \in \mathbb{N}$, such that $\frac{1}{N}<\varepsilon$. Then for each $n \in \mathbb{N}$ such that $n \geq N$ we arrive at $\frac{1}{n} \leq \frac{1}{N}$. Hence $d\left(a, b_{n}\right)<\frac{1}{n} \leq \frac{1}{N}<\varepsilon$. Thus $\lim _{n \rightarrow \infty} d\left(a, b_{n}\right)=0$. By the closeness of $B$ we have $a \in B$.

Lemma 9. Let $(X, d, s)$ be a b-metric space and $A, B \in C B(X)$. Then, for each $\varepsilon>0$ and for all $b \in B$, there exists $a \in A$ such that $d(a, b) \leq H(A, B)+\varepsilon$.

Proof. Let $\varepsilon>0$ and $b \in B$. Suppose that for every $a \in A$ we have $d(a, b)>$ $H(A, B)+\varepsilon$. By the definition of $H$ and the above inequality, we have $H(A, B) \geq$ $d(a, b)>H(A, B)+\varepsilon$, which is a contradiction.

Czerwiks fixed point theorem is the extension of theorem 1 from metric space to b-metric space.

Theorem 10. ([16]) Let $(X, d, s)$ be a complete b-metric space and let $T$ : $X \longrightarrow C B(X)$ be a multi-valued mapping such that $T$ satisfies the inequality

$$
H(T x, T y) \leq a d(x, y)
$$

for all $x, y \in X$, where $0<a<\frac{1}{s^{2}}$. Then $T$ has a fixed point.

The above exploration leads to a doubt that whether there exists a common fixed point for any 2 single-valued mappings and any 2 multi-valued mappings on b-metric space or not. This question inspires us to investigate the existence 
of a common fixed point for multi-valued mapping in complete b-metric space, which is the aim of this research.

\section{Main results}

In this section, we prove the existence of a common fixed point for two multivalued mappings in complete b-metric spaces. In this regard, a relaxing condition for those two mappings is introduced to depict the distinct generalization.

Theorem 11. Let $(X, d, s)$ be a complete b-metric space and let $S, T$ : $X \longrightarrow C B(X)$ be a multi-valued mappings satisfying

$$
H(T x, S y) \leq a d(x, T y)+b(d(x, S y)+d(T y, T x)),
$$

where $a+2 b s<1, a, b \geq 0, b<\frac{1}{s^{2}}$ for all $x, y \in X$. Then $F(T)=F(S) \neq \phi$ and $T x=S x=F(T)$ for all $x \in \stackrel{F}{F}(T)$.

Proof. Fix any $x \in X$ and $0<\varepsilon_{0}<1-b s$. Define $x_{0}=x$ and let $x_{1} \in T x_{0}$. By Lemma(9), we may choose $x_{2} \in S x_{0}$ such that

$$
\begin{aligned}
d\left(x_{1}, x_{2}\right) & \leq H\left(T x_{0}, S x_{0}\right)+\varepsilon_{0} \\
& \leq a d\left(x_{0}, T x_{0}\right)+b\left(d\left(x_{0}, S x_{0}\right)+d\left(T x_{0}, T x_{0}\right)\right)+\varepsilon_{0} \\
& \leq a d\left(x_{0}, x_{1}\right)+b\left(d\left(x_{0}, x_{2}\right)\right)+\varepsilon_{0} \\
& \leq a d\left(x_{0}, x_{1}\right)+b\left(s\left(d\left(x_{0}, x_{1}\right)+d\left(x_{1}, x_{2}\right)\right)\right)+\varepsilon_{0} \\
& \leq a d\left(x_{0}, x_{1}\right)+b s d\left(x_{0}, x_{1}\right)+b s d\left(x_{1}, x_{2}\right)+\varepsilon_{0} \\
d\left(x_{1}, x_{2}\right)-b s d\left(x_{1}, x_{2}\right) & \leq a d\left(x_{0}, x_{1}\right)+b s d\left(x_{0}, x_{1}\right)+\varepsilon_{0} \\
d\left(x_{1}, x_{2}\right)(1-b s) & \leq(a+b s) d\left(x_{0}, x_{1}\right)+\varepsilon_{0} \\
d\left(x_{1}, x_{2}\right) & \leq\left(\frac{a+b s}{1-b s}\right) d\left(x_{0}, x_{1}\right)+\frac{\varepsilon_{0}}{1-b s} \\
d\left(x_{1}, x_{2}\right) & \leq\left(\frac{a+b s}{1-b s}\right) d\left(x_{0}, x_{1}\right)+\varepsilon \quad ; \varepsilon=\frac{\varepsilon_{0}}{1-b s}
\end{aligned}
$$

Similarly, there exists $x_{3} \in T x_{2}$ such that

$$
\begin{aligned}
d\left(x_{3}, x_{2}\right) & \leq H\left(T x_{2}, S x_{0}\right)+\frac{\varepsilon_{0}^{2}}{1-b s} \\
& \leq a d\left(x_{2}, T x_{0}\right)+b\left(d\left(x_{2}, S x_{0}\right)+d\left(T x_{0}, T x_{2}\right)\right)+\frac{\varepsilon_{0}^{2}}{1-b s} \\
& \leq a d\left(x_{2}, x_{1}\right)+b\left(d\left(x_{1}, x_{3}\right)\right)+\frac{\varepsilon_{0}^{2}}{1-b s}
\end{aligned}
$$




$$
\begin{aligned}
& \leq a d\left(x_{2}, x_{1}\right)+b\left(s\left(d\left(x_{1}, x_{2}\right)+d\left(x_{2}, x_{3}\right)\right)+\frac{\varepsilon_{0}^{2}}{1-b s}\right. \\
& \leq a d\left(x_{2}, x_{1}\right)+b s d\left(x_{1}, x_{2}\right)+b s d\left(x_{2}, x_{3}\right)+\frac{\varepsilon_{0}^{2}}{1-b s} \\
d\left(x_{2}, x_{3}\right)-b s d\left(x_{2}, x_{3}\right) & \leq a d\left(x_{2}, x_{1}\right)+b s d\left(x_{1}, x_{2}\right)+\frac{\varepsilon_{0}^{2}}{1-b s} \\
d\left(x_{2}, x_{3}\right)(1-b s) & \leq(a+b s) d\left(x_{1}, x_{2}\right)+\frac{\varepsilon_{0}^{2}}{1-b s} \\
d\left(x_{2}, x_{3}\right) & \leq\left(\frac{a+b s}{1-b s}\right) d\left(x_{1}, x_{2}\right)+\left(\frac{\varepsilon_{0}}{1-b s}\right)^{2} \\
d\left(x_{2}, x_{3}\right) & \leq\left(\frac{a+b s}{1-b s}\right) d\left(x_{1}, x_{2}\right)+\varepsilon^{2}
\end{aligned}
$$

Similarly, there exists $x_{4} \in S x_{2}$ such that

$$
\begin{aligned}
d\left(x_{3}, x_{4}\right) & \leq H\left(T x_{2}, S x_{2}\right)+\frac{\varepsilon_{0}^{3}}{(1-b s)^{2}} \\
& \leq a d\left(x_{2}, T x_{2}\right)+b\left(d\left(x_{2}, S x_{2}\right)+d\left(T x_{2}, T x_{2}\right)\right)+\frac{\varepsilon_{0}^{3}}{(1-b s)^{2}} \\
& \leq a d\left(x_{2}, x_{3}\right)+b\left(d\left(x_{2}, x_{4}\right)\right)+\frac{\varepsilon_{0}^{3}}{(1-b s)^{2}} \\
& \leq a d\left(x_{2}, x_{3}\right)+b\left(s\left(d\left(x_{2}, x_{3}\right)+d\left(x_{3}, x_{4}\right)\right)+\frac{\varepsilon_{0}^{3}}{(1-b s)^{2}}\right. \\
d\left(x_{3}, x_{4}\right)-b s d\left(x_{3}, x_{4}\right) & \leq a d\left(x_{2}, x_{3}\right)+b s d\left(x_{2}, x_{3}\right)+\frac{\varepsilon_{0}^{3}}{(1-b s)^{2}} \\
d\left(x_{3}, x_{4}\right)(1-b s) & \leq(a+b s) d\left(x_{2}, x_{3}\right)+\frac{\varepsilon_{0}^{3}}{(1-b s)^{2}} \\
d\left(x_{3}, x_{4}\right) & \leq\left(\frac{a+b s}{1-b s}\right) d\left(x_{2}, x_{3}\right)+\left(\frac{\varepsilon_{0}}{1-b s}\right)^{3} \\
d\left(x_{2}, x_{3}\right) & \leq\left(\frac{a+b s}{1-b s}\right) d\left(x_{2}, x_{3}\right)+\varepsilon^{3}
\end{aligned}
$$

Continuing this process, we obtain a sequence $\left\{x_{n}\right\}_{n \in \mathbb{N}}$ Therefore,

$$
d\left(x_{n}, x_{n+1}\right) \leq\left(\frac{a+b s}{1-b s}\right) d\left(x_{n-1}, x_{n}\right)+\varepsilon^{n} ; \varepsilon=\frac{\varepsilon_{0}}{1-b s}
$$


For all $n \in \mathbb{N}$, let $k=\frac{a+b s}{1-b s}$. We have

$$
\begin{aligned}
d\left(x_{n}, x_{n+1}\right) & \leq k d\left(x_{n-1}, x_{n}\right)+\varepsilon^{n} \\
& \leq k\left(k\left(d\left(x_{n-2}, x_{n-1}\right)++\varepsilon^{n-1}\right)+\varepsilon^{n}\right. \\
& =k^{2} d\left(x_{n-2}, x_{n-1}\right)+k \varepsilon^{n-1}+\varepsilon^{n} \\
& \leq k^{2}\left(k\left(d\left(x_{n-3}, x_{n-2}\right)+\varepsilon^{n-2}\right)+k \varepsilon^{n-1}+\varepsilon^{n}\right) \\
& =k^{3} d\left(x_{n-3}, x_{n-2}\right)+k^{2} \varepsilon^{n-2}+k \varepsilon^{n-1}+\varepsilon^{n} \\
& \leq \cdots \\
& \leq k^{n} d\left(x_{0}, x_{1}\right)+\sum_{r=1}^{n} k^{n-r} \varepsilon^{r}
\end{aligned}
$$

This shows that

$$
\begin{aligned}
& d\left(x_{n}, x_{n+1}\right) \leq k^{n} d\left(x_{0}, x_{1}\right)+\sum_{r=1}^{n} k^{n-r} \varepsilon^{r} \\
& \sum_{n=1}^{N} d\left(x_{n}, x_{n+1}\right) \leq \sum_{n=1}^{N} k^{n} d\left(x_{0}, x_{1}\right)+\sum_{n=1}^{N}\left(\sum_{r=1}^{n} k^{n-r} \varepsilon^{r}\right) \\
& =\sum_{n=1}^{N} k^{n} d\left(x_{0}, x_{1}\right)+\sum_{n=1}^{N}\left(\sum_{r=0}^{n-1} k^{r} \varepsilon^{n-r}\right) \\
& =\sum_{n=1}^{N} k^{n} d\left(x_{0}, x_{1}\right)+\sum_{n=1}^{N} \varepsilon^{n}\left(\sum_{r=0}^{n-1} k^{r}\right) \\
& =d\left(x_{0}, x_{1}\right) \sum_{n=1}^{N} k^{n}+\sum_{n=1}^{N} \varepsilon^{n}\left(\frac{1\left(1-k^{n}\right)}{1-k}\right) \\
& <d\left(x_{0}, x_{1}\right) \sum_{n=1}^{N} k^{n}+\sum_{n=1}^{N} \varepsilon^{n}\left(\frac{1}{1-k}\right) \\
& =d\left(x_{0}, x_{1}\right) \sum_{n=1}^{N} k^{n}+\left(\frac{1}{1-k}\right) \sum_{n=1}^{N} \varepsilon^{n} \\
& \sum_{n=1}^{\infty} d\left(x_{n}, x_{n+1}\right)=d\left(x_{0}, x_{1}\right) \sum_{n=1}^{\infty} k^{n}+\left(\frac{1}{1-k}\right) \sum_{n=1}^{\infty} \varepsilon^{n} \\
& =d\left(x_{0}, x_{1}\right)\left(\frac{k}{1-k}\right)+\left(\frac{1}{1-k}\right)\left(\frac{\varepsilon}{1-\varepsilon}\right) \\
& =\frac{1}{1-k}\left[k d\left(x_{0}, x_{1}\right)+\frac{\varepsilon}{1-\varepsilon}\right]<\infty
\end{aligned}
$$


Hence we get $\lim _{n \rightarrow \infty} d\left(x_{n}, x_{n+1}\right)=0$.

Now, we show that $\left\{x_{n}\right\}$ is Cauchy sequence in $X$.

Let $m, n>0$ with $m>n$, as $m=n+p ; p \in \mathbb{N}$

$$
\begin{aligned}
d\left(x_{n}, x_{m}\right) & =d\left(x_{n}, x_{n+p}\right) \\
& \leq s d\left(x_{n}, x_{n+1}\right)+s^{2} d\left(x_{n+1}, x_{n+2}\right)+\ldots+s^{p} d\left(x_{n+p-1}, x_{n+p}\right)
\end{aligned}
$$

when we take $n \rightarrow \infty$, we get $\lim _{n \rightarrow \infty} d\left(x_{n}, x_{m}\right)=0$.

Hence the sequence $\left\{x_{n}\right\}$ is a Cauchy sequence since $(X, d, s)$ is complete, there exists $z \in X$ such that $x_{n} \rightarrow z, \lim _{n \rightarrow \infty} d\left(x_{n}, z\right)=0$.

Now, we show that $z$ is a fixed point of $T, S$. We have

$$
\begin{aligned}
d(z, T z) \leq & s\left(d\left(z, x_{2 n+2}\right)+d\left(x_{2 n+2}, T z\right)\right) \\
\leq & s\left(d\left(z, x_{2 n+2}\right)+H\left(S x_{2 n}, T z\right)\right) \\
\leq & s\left(d\left(z, x_{2 n+2}\right)+H\left(T z, S x_{2 n}\right)\right) \\
\leq & s\left(d\left(z, x_{2 n+2}\right)+a d\left(z, T x_{2 n}\right)+b\left(d\left(z, S x_{2 n}\right)+d\left(T x_{2 n}, T z\right)\right)\right. \\
\leq & s\left(d\left(z, x_{2 n+2}\right)+a d\left(z, x_{2 n+1}\right)+b\left(d\left(z, x_{2 n+2}\right)+d\left(x_{2 n+1}, T z\right)\right)\right. \\
\leq & s\left(d\left(z, x_{2 n+2}\right)+a d\left(z, x_{2 n+1}\right)+b d\left(z, x_{2 n+2}\right)\right. \\
& \left.+b\left(s\left(d\left(x_{2 n+1}, z\right)+d(z, T z)\right)\right)\right)
\end{aligned}
$$

Letting $n \rightarrow \infty$ in the inequality above, we obtain $d(z, T z) \leq s^{2} b d(z, T z)$ then we have $d(z, T z)=0$. Thus $z \in T z$. We get $F(T) \neq \phi$.

Next, we show that $T z=S z$.

$$
\begin{aligned}
H(T z, S z) & \leq a d(z, T z)+b(d(z, S z)+d(T z, T z)) \\
& \leq b d(z, S z) \\
& \leq b H(T z, S z) \\
H(T z, S z)-b H(T z, S z) & \leq 0 \\
H(T z, S z)(1-b) & \leq 0 \\
H(T z, S z) & \leq 0 \\
H(T z, S z) & =0
\end{aligned}
$$

Thus $T z=S z$. We get $F(S) \neq \phi$.

We arrive at the proof of our final result which require the following steps: 1.F $(T)=T z$

$2 . S x=T x$ for all $x \in F(T)$ 
3. $F(T)=F(S)$

First we will show that $F(T)=T z$.

Let $x \in F(T)$, we have $x \in T x$,

Proof.

$$
\begin{aligned}
d(x, T z) & \leq H(T x, T z) \\
& =H(T x, S z) \\
& \leq a d(x, T z)+b(d(x, S z)+d(T z, T x)) \\
& \leq a d(x, T z)+b d(x, S z)+b d(T z, T x) \\
& \leq a d(x, T z)+b d(x, T z)+b d(T z, x) \\
d(x, T z) & \leq(a+2 b) d(x, T z) \\
d(x, T z)-(a+2 b) d(x, T z) & \leq 0 \\
d(x, T z)(1-(a+2 b)) & \leq 0 \\
d(x, T z) & \leq 0 \\
d(x, T z) & =0
\end{aligned}
$$

Thus $x \in T z$, we get $T x \subseteq T z$.

Therefore $F(T) \subseteq T z$.

Let $x \in T z$. We show that $x \in T x$.

$$
\begin{aligned}
d(x, T x) & \leq H(T z, T x) \\
& =H(S z, T x) \\
& =H(T x, S z) \\
& \leq a d(x, T z)+b(d(x, S z)+d(T z, T x)) \\
& \leq a d(x, T z)+b d(x, S z)+b d(T z, T x) \\
& \leq a d(x, T z)+b d(x, T z)+b d(x, T x) \\
d(x, T x)-b d(x, T x) & \leq 0 \\
d(x, T x)(1-b) & \leq 0 \\
d(x, T x) & \leq 0 \\
d(x, T x) & =0
\end{aligned}
$$

Thus $x \in T x$, we get $T z \subseteq T x$

therefore $T z \subseteq F(T)$.

Hence $F(T)=T z$. 
Next, we show that $T x=S x$.

For all $x \in F(T)$,

$$
\begin{aligned}
H(T x, S x) & \leq a d(x, T x)+b(d(x, S x)+d(T x, T x)) \\
& \leq a d(x, T x)+b d(x, S x)+b d(T x, T x) \\
& \leq b d(x, S x) \\
& \leq b H(T x, S x) \\
H(T x, S x)-b H(T x, S x) & \leq 0 \\
H(T x, S x)(1-b) & \leq 0 \\
H(T x, S x) & \leq 0 \\
H(T x, S x) & =0
\end{aligned}
$$

Thus $T x=S x$, for all $x \in F(T)$.

Now, we show that $F(T)=F(S)$.

Let $x \in F(T)$, that is $x \in T x$ by previous result, we have $T x=S x$.

thus $x \in S x$ which means that $x \in F(S)$.

So we automatically get $F(T) \subseteq F(S)$.

It remains to show that $F(S) \subseteq F(T)$.

Let $x \in F(S)$,

$$
\begin{aligned}
d(x, T x) & \leq H(S x, T x) \\
& =H(T x, S x) \\
& \leq a d(x, T x)+b(d(x, S x)+d(T x, T x)) \\
& \leq a d(x, T x)+b d(x, S x)+b d(T x, T x) \\
d(x, T x) & \leq a d(x, T x) \\
d(x, T x)-a d(x, T x) & \leq 0 \\
d(x, T x)(1-a) & \leq 0 \\
d(x, T x) & \leq 0 \\
d(x, T x) & =0
\end{aligned}
$$

Thus $x \in T x$, we get $F(S) \subseteq F(T)$.

Hence $F(T)=F(S)$. 
Hence $F(T)=F(S) \neq \phi$ and $S x=T x=F(T)$ for all $x \in F(T)$, which complete the proof.

The following corollaries can be deduced as particular cases of the main theorem.

Corollary 12. Let $(X, d)$ be complete metric space and let $S, T: X \longrightarrow$ $C B(X)$ be a multi-valued mappings satisfying

$$
H(T x, S y) \leq r d(x, T y)
$$

where $r \in[0,1)$ for all $x, y \in X$. Then $F(T)=F(S) \neq \phi$ and $T x=S x=F(T)$ for all $x \in F(T)$.

Proof. Take $b=0$ and $a=r$ in Theorem 11 then we get desired result.

Corollary 13. Let $(X, d)$ be a complete metric space, and let $T, S$ be self-map on $X$ and if there exists $r \in[0,1)$ such that

$$
d(T x, S y) \leq r d(x, T y)
$$

for all $x, y \in X$. Then $S$ and $T$ have a unique common fixed point.

Proof. It follows by applying Corollary 12 and the fact that $H(T x, S y)=$ $d(T x, S y)$.

Remark 14. (a) Corollary 12. is actually a result on complete metric spaces proved by Lai-Jiu Lin and Sung-Yu Wang [8]. We can say that Theorem 11 is a generalization of Lai-Jiu Lin and Sung-Yu Wang [8] in the framework of b-metric spaces.

(b) Corollary 12 in the case of single-valued mappings $T, S: X \rightarrow X$ is conducted as in Corollary 13.

Corollary 15. Let $(X, d)$ be complete metric space and let $T: X \longrightarrow$ $C B(X)$ be a multi-valued mappings with nonempty compact valued such that

$$
H\left(T x, T^{2} y\right) \leq r d(x, T y)
$$

where $r \in[0,1)$ for all $x, y \in X$. Then $F(T) \neq \phi$ and $T x=F(T)$ for all $x \in F(T)$.

Proof. Take $S=T^{2}$ with $b=0$ and $a=r$ in Theorem 11 and complete the proof. 


\section{Conclusion}

In this paper, we have proved some common fixed point results for any two multi-valued mappings on complete b-metric spaces. The main theorem is an extension of the common fixed point theorem for single- value mappings on complete b-metric spaces. In addition the theorem can be regarded as a generalization of some previous results on complete metric spaces. Assuming specific values for the constants in the main theorem yields the consequence corollaries, which are the recent results from the current literature. The way of proof can be developed for further investigation on more extensive spaces.

\section{Acknowledgements}

The authors would like to thank Faculty of Science Burapha University for financial support. The authors are also grateful to the reviewers for comments that greatly improved the manuscript.

\section{References}

[1] S. Banach, Sur les operations dans les ensembles abstraits et leur applications aux equations integrales, Fundamenta Mathematicae, 3 (1922), 133-181.

[2] R. Kannan, Some results on fixed points, Bull. Calcutta Math. Soc., 60 (1968), 71-76.

[3] R. Kannan, Some results on fixed points-II, Amer. Math. Monthly., 76, No.4 (1969), 405-408, doi: 10.2307/2316437.

[4] W.A. Kirk, P.S. Srinivasan, P. Veeramani, Fixed points for mappings satisfying cyclical contractive conditions, Fixed Point Theory, 4, No.1 (2003), 79-89.

[5] S.B. Nadler, Multivalued contraction mappings, Pacific Journal of Mathematics, 30, No. 2 (1969), 475-488.

[6] P. Srivastava, V.K. Gupta, A note on common fixed points, Yokohama Math.J., 19 (1971), 91-95.

[7] J. Jungck, Conmuting mappings and fixed points, Amer. Math. Monthly., 83, No.4 (1976), 63-261, doi: 10.2307/2318216.

[8] L.J. Lin, S.Y. Wang, Common Fixed Point Theorems for a Finite Family of Discontinuous and Noncommutative Maps, Fixed Point Theory and Applications, (2011), 9 pages, doi: $10.1155 / 2011 / 847170$.

[9] S. Czerwik, Contraction mappings in b-metric spaces, Acta Math. Inform. Univ. Ostrav., 1 (1993), 5-11.

[10] H. Aydi, M.F. Bota, E. Karapinar, S. Mitrovic, A fixed point theorem for set-valued quasicontractions in b-metric spaces, Fixed Point Theory Appl., (2012), 8 pages, doi: 10.1186/1687-1812-2012-88. 
[11] M. Bota, Dynamical aspects in the theory of multivalued operators PhD thesis, (n.d).

[12] S. Reich, Some problems and results in fixed point theory, Contemporary Mathematics, 21 (1983), 179-187, doi: 10.1090/conm/021.

[13] N. Mioguchi, W. Takahashi, Fixed point theorems for multivalued mappings on complete metric spaces, Journal of Mathematical Analytic and Applications, 141 (1989), 172-188, doi: 10.1016/0022-247X(89)90214-X.

[14] S. Czerwik, Nonlinear set-valued contraction mappings in b-metric spaces, Atti Semin. Mat. Fis. Univ. Modena., 46, No.3 (1998), 263-276.

[15] M. Boriceanu, M. Bota, A. Petrusel, Multivalued fractals in b-metric spaces, Cent. Eur. J. Math., 8 (2010), 367-377, doi: 10.2478/s11533-010-0009-4.

[16] S. Czerwik, K. Dlutek, S.L. Singh, Round-off stability of iteration procedures for operators in b-metric spaces, J. Natur. Phys. Sci., 11 (1997), 87-94. 
